\title{
Digital Model for Electrocardiogram
}

\author{
M.R.S. Reddy ${ }^{1}$ \\ Department of Electrical Engineering \\ Indian Institute of Science, Bangalore, India.
}

\begin{abstract}
The electrophysiology of the heart is represented by a Linear Time Invariant (LTI) system function to represent the electrocardiogram (ECG) in an efficient way. The ECG as well as its component waves, $P, Q R S$ and $T$, are independently subjected to Discrete Cosine Transform (DCT) and transform outputs are modelled using sixth order system for complete ECG and second order system for each component wave. When the complete ECG model is decomposed into three second order subsystems connected in parallel, denominator polynomials of these subsystems are closely matching with the denominator polynomials of three second order models derived for component waves. Sum of the component models impulse response is in good agreement with that of the ECG model. A relation between system parameters and the time domain signal peaks is presented. Validity of the method is discussed for the ECGs of bizarre shape and more than one ECG cycle in a sample frame is considered for modelling. The model provides a method to analyse and synthesise ECGs of different morphologies.
\end{abstract}

I. Introduction

Electrocardiogram (ECG) is a non-invasive, widely used diagnostic tool in clinical circles to determine the cardiac function. Many analysis methods based on time as well as frequency domain information of ECG are reviewed in [1]. However, representation of the ECG as a system output, like electroencephalogram (EEG) [2], electromyogram (EMG) [3], speech [4] etc. is highly relevant for a better understanding of the function of heart. The field theory approach tried to correlate atrial and ventricular activities to ECG signal by defining forward and inverse problems [5]. Due to computational complexity and nonlinearity of human body this approach could not yield simple solution for ECG analysis. Cadzow and Hwang[6] have represented the Discrete Cosine Transform (DCT) of ECG as a rational polynomial and used it for data compression. The system models derived by Murthy and Rangaraj [7] for minimum and maximum phase correspondents of ECG are of higher order (20 to 25) and did not give explicit relation between ECG and system parameters. The present paper describes a method of representing complete ECG cycle with a Linear Time Invariant (LTI) system of much lower order compared to [7] ,

\footnotetext{
1 Presently working as a visiting scientist at The Department of Clinical Physiology, University of Lund, Lund, Sweden.
}

and also decomposition of ECG model into three subsystems corresponding to the three component waves $\mathrm{P}, \mathrm{QRS}$ and $\mathrm{T}$ of the ECG. Performance of the method for different morphological ECG representation and relation between time domain signal peaks and system perameters are discussed.

The second section derives an analogy between electrophysiology and LTI system and presents a system model for efficient representation of ECG. Details of manual delineation of component waves, transformation and modelling of components and complete ECG cycle are explained in section III. Results and discussion are presented in section IV and the final section is devoted to conclusions.

\section{Digital model for ECG Signal}

Normally a cardiac cycle is initiated by a pacemaking pulse from the Sinoatrial (SA) node and consists of three distinct electrical components $\mathrm{P}, \mathrm{QRS}$ and $\mathrm{T}$ waves representing depolarisation and repolarisation of atrial and ventricular muscle in the heart. The amplitude, shape and inter component distances will describe some aspects of the function of the heart. To translate this activity into mathematical parameters the ECG has to be represented suitably with a system function.

From what has been said above, the following salient points can be deduced on the electrophysiology of the heart: (i) the excitation pulse from the SA node can be approximated as an impulse relative to the response time of the excited system - the cardiac muscles, (ii) the excitation source (SA node) is distinct from the excited system, (iii) the events $P$, QRS and T are the result of depolarisation of atria, ventricles, and repolarisation of ventricles. This behaviour of the system can be represented by a LTI system function $\mathrm{H}(\mathrm{z})$ excited by a train of impulses. In this study the output of LTI system is assumed as the Discrete Cosine Transformed ECG rather than direct time domain signal. This procedure resulted in a system function of much lower order compared to $[6,7]$ and linearity property of DCT enabled to decompose the ECG system function into three component subsystems connected in parallel as shown in block diagram Fig. 1. For this model, the 
excitation source generates a train of impulses and the excited system is conceived to be made up of a combination of three independent parallel subsystems $H_{i}(z), i=p, q, t$, each excited by an impulse. Having chosen the source as an impulse generator, the parameters of the excited subsystems are to be chosen such that the DCT of $P, Q R S$ and $T$ waves emerge as the output of subsystems $p, q$ and $t$ respectively. So, the subsystems impulse responses are added and subjected to InverseDCT (IDCT) to realise complete ECG cycle. When subsystems impulse responses are subjected to IDCT, the time domain $\mathrm{P}, \mathrm{QRS}$ and $\mathrm{T}$ waves resulted. Thus, proposed model $H(z)$ in Fig. 1. is the sum of subsystems $H_{p}(z), H_{q}(z)$ and $\mathrm{H}_{\mathrm{t}}(\mathrm{z})$, that represent the component waves $\mathrm{P}, \mathrm{QRS}$ and $\mathrm{T}$ of ECG cycle. That is

$$
H(z)=H_{p}(z)+H_{q}(z)+H_{t}(z)
$$

\section{Method}

For this study a set of 500 ECGs of different morphologies are selected from the MIT database and a database collected at the Indian Institute of Science, India. Both databases are acquired by subjecting the ECG to a low pass filter with cut off frequency at $125 \mathrm{~Hz}$ and digitised with 12 bit resolution $\mathrm{ADC}$ at 500 samples per second. To demonstrate the system decomposition, a normal lead II ECG cycle is chosen and the P-QRS and QRS-T junction points are manually identified on the baseline. $P$ wave component is defined from beginning of ECG cycle up to the previous sample of $\mathrm{P}-\mathrm{QRS}$ junction, QRS component is considered from $\mathrm{P}$-QRS junction point to the previous sample of QRS-T junction and $\mathrm{T}$ component is considered from QRS-T junction point to the end of ECG cycle. End points of each component are repeated suitably and lengths and starting points of all components and complete ECG cycle are made equal. This operation facilitated the application of DCT on equal length signals. From the linearity property of DCT, the sum of component wave DCTs resulted in the DCT of complete ECG cycle. In

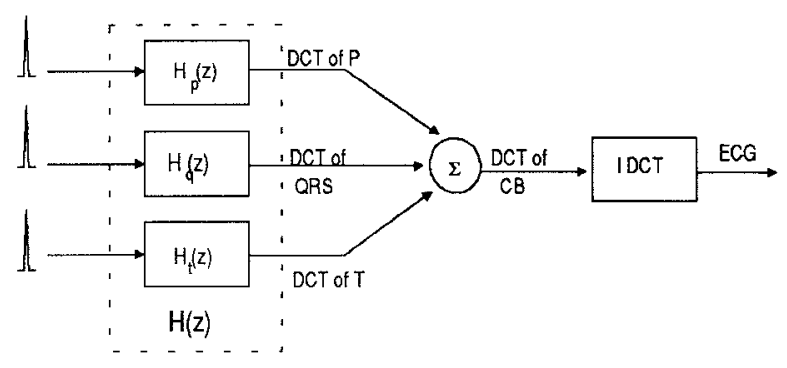

Fig.1. Block dagram of Digital Model for ECG this case signal value on the base line is assumed as zero, otherwise a small offset value has to be added depending on the values of P-QRS and QRS-T junction points. The transformed component waves and complete cycle of ECG are separately modelled using an iterative modelling procedure of Steiglitz McBride method [8] with second order systems for components and sixth order system for complete cycle. PoleZero positions in the z-plane derived for complete ECG as well as component models are shown in Table 1 . To evaluate the performance of the method Normalised mean square error (NMSE) is calculated between the time domain signal and the IDCT of model output.

\section{Results and Discussion}

The model provides accurate signal representation with fewer number of parameters and establishes a relationship between the model poles-zeros and signal components. From Table 1. it can be noticed that, three poles of complete ECG model form three clusters in the z-plane with a zero separating each cluster. Further it was shown [9] that the angle $\Theta$ subtended at the origin by each complex pole corresponds to a time domain ECG component peak sample number by the relation

$$
\theta=((2 n+1) 180) / 2 N
$$

$\mathrm{n}=$ component peak sample number and

$\mathrm{N}=$ number of samples used for modelling.

When the complete ECG system function is decomposed in to

Table 1. Pole-Zero locations in z-plane for Models of Complete ECG cycle and Component waves.

\begin{tabular}{ccccc} 
& \multicolumn{2}{c}{ Poles } & \multicolumn{2}{c}{ Zeros } \\
& radius & angle & radius & angle \\
ECG & 0.7229 & 35.73 & 0.3105 & 0.00 \\
cycle & 0.9782 & 65.87 & 0.7358 & 40.49 \\
& 0.7527 & 147.60 & 0.8062 & 74.49 \\
& & & 0.8712 & 180.00 \\
P & 0.9286 & 26.39 & 0.0042 & 0.00 \\
Wave & & & 0.7524 & 0.00 \\
& & & & \\
QRS & 0.9798 & 67.09 & 0.4474 & 0.00 \\
Complex & & & 0.0743 & 0.00 \\
& & & & \\
T & 0.7538 & 146.20 & 0.2861 & 0.00 \\
Wave & & & 0.8831 & 180.0
\end{tabular}


three second order subsystems connected in parallel, each subsystem results with one complex pole of the complete ECG system. The last six rows of Table 1. show pole-zero locations of three component models. Pole locations of QRS and T models are in good agreement with pole locations of subsystems derived from the complete ECG system. Though a small deviation can be noticed in $\mathrm{P}$ wave model pole, it is still with in the boundary of its cluster.

Analysis of different ECG morphologies show that higher orders $(10,10)$ or $(12,12)$ are required to model abnormal beats of bizarre type. In this case the subsystem order of bizarre component wave will increase by two or four depending on morphology of component, but pole(s) of each component wave subsystem will form different clusters. The distances between clusters depend on inter component distances. When more than one ECG cycle is present in the frame considered for modelling the clustering of component poles behave in the same way as they did in the case of a single beat, but the distance between clusters will be scaled according to the number of sample considered for modelling. When a random noise of peak to peak amplitude equal to $25 \%$ of QRS complex amplitude is added to the clean ECG signal and modelled, the model output is in good agreement with the ECG signal. This reveals that the method seems to model only the global features of given signals resulting higher error for noisy signals although the global fit between the signal and its model may be quite satisfactory. In each case the NMSE is calculated and this figure was less than $10 \%$ for clean ECGs and an increase of up to $10 \%$ depending on the noise level in the signal. Convergence problem will arise if model order is increased beyond what is required for a given signal. When the model order used deviates from the actual one by $\pm(2,2)$ the method is not affected as such. When the order is underestimated a small component may be lost resulting an increase in error.

\section{v. Conclusions}

A new digital model is proposed for the generation of ECG signal from a system theoretic point of view. The component waves $P, Q R S$ and $T$ of the ECG. signal are considered as three independent events and each component wave is approximated with a system function of suitable order. For the purpose of modelling, the discrete cosine transformed (DCT) ECG signal/ transformed components is/ are made use of instead of the direct time signal/components. Thus the impulse response of each subsystem is considered as the discrete cosine transformed version of the respective component wave. The inverse transform of the impulse response will give the time domain signal. All the subsystems are combined in parallel so that the complete ECG cycle is realised as the output of the overall system function.

\section{Acknowledgments}

Author would like to acknowledge late Prof. I.S.N. Murthy, the Department of Electrical Engineering, Indian Institute of Science, India, for inspiring and starting the work and the Department of Clinical Physiology, University of Lund, Sweden, for providing funds for the preparation of this paper.

References

1. O. Pahlm and L. Sormmo, Software QRS detection in ambulatory monitoring - a review', Med. Biol. Eng., Vol. 23, pp. 289-297, 1984.

2. L.H. Zetterberg, 'Estimation of parameters for linear difference equation with application to EEG analysis', Math. Biosci., Vol. 5, pp. 227-275, 1969.

3. O. Paiss and G.F. Inbar, 'Autoregressive modelling of surface EMG and its spectrum with application to fatigue', IEEE Trans. Biomed. Eng., Vol. 34, pp. 761-770, 1987.

4. J. Makhoul, 'Linear predictioon: A tutorial review', Proc. IEEE Vol. 63, pp $561-580,1975$.

5. Y. Kim and W.J. Tompkins, 'Forward and inverse high frequency electrocardiography', Med. Biol. Engg., Vol-19, pp 11-22, 1981.

6. J. Cadzow and T.T. Hwang, 'Signal representation: An efficient procedure', IEEE Trans. Acoust. Speech, Signal Processing, Vol. -25, pp 461-466, 1977.

7. I.S.N. Murthy and M.R. Rangaraj, 'New concepts for PVC detection', IEEE Trans. Biomed. Eng., Vol. -26, pp 330-344, 1979.

8. K. Steiglitz and L.E. McBride, 'A technique for the identification of linear systems', IEEE Trans. Automatic Control, pp 461-464, 1965.

9. M. Ramasubba Reddy, 'Digital Models for Analysis and Synthesis of ECG', Ph.D. Thesis, Dept. of Electrical Eingg., Indian Institute of Science, India, 1990. 